\title{
ISDN-Abschaltung
}

\section{Wie Ärzte jetzt einen Telefonausfall verhindern}

\author{
2018 ist Schluss mit dem ISDN-Netz. Dann wollen große Anbieter ihre \\ Leitungen endgültig umstellen. Aber gerade Arztpraxen kann es auch \\ schon früher treffen. Was können Betroffene jetzt tun?
}

$\mathrm{D}$ as digitale Telefonnetz ISDN (Integrated Services Digital Network) war zuverlässig, lieferte gute Sprachqualität und unterstützte praktische Komfortfunktionen. Deshalb entschieden sich auch viele Praxen, Kliniken und Ärztehäuser für diese Anschlüsse. Nun wollen die Telekommunikationsanbieter ISDN loswerden. Spätestens 2018 plant die Telekom, die letzten alten Anschlüsse abgeschaltet zu haben. Auch Telekom-Wiederverkäufer wie 1\&1 sind betroffen. Nur Vodafone will seinen Geschäftskunden notfalls noch ein paar Jahre länger Zeit lassen. Für die Anbieter werden Betrieb und Unterhalt der ISDN-Technik immer teurer. Sie möchten die Kundenanschlüsse deshalb lieber auf die günstigere und modernere IP-Technik umstellen.

\section{Umstellung läuft auf vollen Touren}

Der neue Anschlusstyp nutzt zum Telefonieren das Internet-Protokoll (IP). Mit zwei Leitungen und drei bis zehn Rufnummern bietet er vergleichbaren Komfort wie ISDN und während IP-Telefonie früher fehleranfällig und qualitativ minderwertig war, ist die Technik mittlerweile deutlich gereift. Die Umstellung läuft daher bereits auf vollen Touren. Viele ISDN-Kunden wurden von ihrem Anbieter bereits angeschrieben und zum Wechsel gedrängt. Um komplette Vermittlungsstellen - also alle Kunden eines Stadtviertels beziehungsweise einer Region - zügig auf "All-IP“ umstellen zu können, drohen die Betreiber verbliebenen Verweigerern im schlimmsten Fall mit der Zwangskündigung des An- schlusses. Deshalb sollten sich Betroffene nicht allzu viel Zeit lassen, sich auf die neue Sachlage einzustellen.

Wie man angesichts der IP-Umstellung am besten vorgehen sollte, hängt von der bislang vorhandenen Telefontechnik ab. Größere Praxen oder Kliniken, die eine ausgewachsene Nebenstellenanlage betreiben, wenden sich am besten an den Anbieter oder Betreuer dieser Systeme. In den meisten Fällen muss nicht die komplette Telefonanlage ausgewechselt werden. Oft genügt es, die Komponente zu modernisieren, die bisher den Anschluss ans ISDN verwaltete. Doch neben den Telefonanschlüssen versorgen ISDN-Leitungen oft auch weitere Systeme wie EC-Bezahlterminals, Alarmanlagen oder Aufzug-Notrufsysteme. Auch dann gilt: Der Ansprechpartner ist der jeweilige Anbieter.

\section{All-IP - was ist das?}

Der Begriff bezeichnet die Umstellung bisheriger Übertragungstechniken in Telekommunikationsnetzen auf die Basis des Internet-Protokolls (IP) - also vom analogen auf den rein digitalen Anschluss.

Einzelne Dienste wie Telefonie, Fax, Mobilfunk, Internet oder Fernsehen werden dabei einheitlich über die digitalen Leitungen geschickt.

Der Nachteil: Fällt die DSL-Leitung aus oder der Router geht kaputt oder hat eine Störung, dann schweigt auch das Telefon.
Einzelpraxen und kleine Praxisgemeinschaften haben jedoch in der Regel keine Telefonanlage. Ihnen genügen häufig ein bis zwei ISDN-Anschlüsse mit zwei bis vier Außenleitungen. Dementsprechend gibt es in solchen Fällen oft auch kein Wartungsunternehmen, das für die Technik zuständig wäre. Dann gilt häufig das Motto „Hilf Dir selbst“. Auch wenn die externe Telefonleitung auf IP-Technik umgestellt wird, müssen die internen Telefone und andere Geräte wie Fax oder Anrufbeantworter in der Regel nicht ersetzt werden. Benötigt wird vielmehr ein Adapter, der zwischen der intern genutzten, vorhandenen ISDN-Technik und der künftigen externen IP-Leitung ,übersetzt“. Diese Funktion übernehmen nicht zuletzt moderne InternetRouter, beispielsweise die "Fritzboxen“ von AVM. Anbieter wie 1\&1 und Vodafone liefern dieselben AVM-Router zum Teil unter eigenen Produktbezeichnungen. Nicht so einfach ist der Umstieg bei den Speedport-Routern der Telekom. Sie verfügen nur über analoge Telefonschnittstellen. Mit dem separat angebotenen Speedport ISDN-Adapter lassen sich aber auch diese Router erweitern.

\section{Anbieter an Kosten beteiligen}

Wenn durch die IP-Umstellung Kosten für Neuanschaffungen oder Anpassungen anfallen, sollte man auf jeden Fall den Netzbetreiber darauf ansprechen. Zumindest anteilig werden solche Kosten im Rahmen der Kundenpflege teilweise übernommen. Oft steht nach der Umstellung sogar etwas mehr Telefonkomfort zur Verfügung als vorher. Bei Bedarf lassen sich an den Internet-Boxen sogar schnurlose Telefone anmelden. Im Zusammenspiel mit geeigneten Endgeräten sowie Gegenstellen lässt sich unter Umständen sogar die neue „HD-Telefonie“ nutzen.

Hannes Rügheimer 